\title{
Physical Activity Behaviour: An Overview of Current and Emergent Theoretical Practices
}

\author{
Duncan S. Buchan, ${ }^{1}$ Stewart Ollis, ${ }^{1}$ Non E. Thomas, ${ }^{2}$ and Julien S. Baker ${ }^{1}$ \\ ${ }^{1}$ Health and Exercise Sciences, School of Science and Technology, University of the West of Scotland, Hamilton ML3 OJB, UK \\ ${ }^{2}$ School of Human Sciences, Swansea University, Swansea SA2 8PP, UK \\ Correspondence should be addressed to Duncan S. Buchan, duncan.buchan@uws.ac.uk
}

Received 21 November 2011; Revised 30 March 2012; Accepted 10 May 2012

Academic Editor: Amy A. Gorin

Copyright (C) 2012 Duncan S. Buchan et al. This is an open access article distributed under the Creative Commons Attribution License, which permits unrestricted use, distribution, and reproduction in any medium, provided the original work is properly cited.

\begin{abstract}
Physical activity research has been dominated by traditional cognitive rationale paradigms utilized within other domains. Though this approach to physical activity behavior has greatly enhanced our understanding of the key determinants, it has done little to eradicate the health problems we currently face. In order to achieve lasting change though, multilevel interventions may prove effective. Ecological perspectives have been proposed as an effective approach in combating current physical inactivity levels. Nevertheless, this approach is in its infancy and much has still to be learned. The aim of this paper is to provide an overview of the main behavioral models used within the physical activity domain while proposing the need for further models that will embrace the principles presented by ecological and complexity theories.
\end{abstract}

\section{Introduction}

The World Health Organization (WHO) rates physical inactivity as one of the main causes of premature death in developed countries, implicated in the aetiology of many chronic diseases such as cancer, cardiovascular disease, diabetes, and obesity [1]. Though the beneficial effects of physical activity (PA) on health are well known and firmly established, few are meeting current PA recommendations [2]. It is now appreciated that individuals face considerable barriers when changing complex behaviors such as PA [3]. Though early endeavours into the promotion of PA tended to be largely atheoretical, the need for interventions to be informed through appropriate theoretical underpinning and allow subsequent replication was essential. This paradigm shift saw subsequent authors focusing towards understanding the determinants and correlates of $\mathrm{PA}$, in particular psychosocial influences [4]. As such, theories of behavioural change initially developed within social psychology have dominated the literature.

Though some models have been applied more frequently than others, the four most prominent theories utilized within a PA context are The Social Cognitive Theory (SCT), The
Theory of Planned Behavior (TPB), The Self-Determination Theory (SDT), and The Transtheoretical Model (TTM) [5]. Undoubtedly the application of these theories has greatly enhanced our understanding of the psychological influences and processes which influence PA behaviour. Even so, this reliance upon traditional cognitive rationale paradigms has done little to eradicate current health problems.

What has become apparent within the last decade is that changing behavior is a complex and multifaceted phenomenon with multiple levels of influences. Thus, in order to achieve enduring changes in behavior multilevel interventions that focus on targeting individuals, social environments, physical environments, and policies have been proposed [6]. Since social ecological models of health behaviour focus on individual, as well as social, policy, and environmental influences $[7,8]$, researchers have recently embraced the use of such frameworks to inform interventions. It seems that, once again, the PA domain is at the tipping point of another paradigm shift.

Despite ecologically based multilevel interventions holding great potential in changing complex behaviors, certain issues associated with such an approach needs to be considered. For instance, though guiding frameworks such 
as the social ecological theory are useful for considering behavioural determinants broadly, there is a lack of models available that provide specific mechanisms through which particular influences may interact and influence behavior. This is to be expected given that much research is still needed before an agreed consensus regarding the factors that exert the most influence on PA behavior, in a specific context, is formed. As the TTM and the TPB provide mechanisms and variables to target for influencing behavior, it is unsurprising that researchers have tended to rely heavily upon these theories when devising interventions. Thus, the purpose of this paper is twofold. First, a very brief critique of four popular cognitive-based theories used within the PA domain will be presented. Secondly, attention will be given to the emergent ecological models currently being proposed with further research directions being offered.

\section{Current Theoretical Practice within Physical Activity}

The two distinct approaches currently dominating the literature are the staged-based approach and the cognitivebased approach [4]. Whereas stage-based models propose that individuals go through stages in order to adopt or maintain complex behaviors like PA, cognitive-based approaches assume that complex behaviors are controlled by rationale cognitive activity. Within this approach interventions focus on indentifying determinants that can explain behavior. At this point it is important to recognize that other popular models exist including the Sports Commitment Model [9], the Schema Theory, the Psychological Continuum Model, the Social Support Model, the Enjoyment Model, and the Health Belief Model [6]. However, the TPB, the SCT, the SDT, and The TTM models represent those which have been tested and adopted most widely in the health behavior and PA literature [10]. Therefore it is felt that these four models are most worthy of attention and will be discussed within this brief paper.

\section{The Social Cognitive Theory within Physical Activity}

A widely used theoretical model of behavior change favoured by researchers is the SCT [11]. This model describes factors that may affect and determine behavior while also specifying mechanisms through which these factors work and how they may be altered into effective health behaviors. The structure and predictive utility of the SCT has been tested across numerous domains and populations $[12,13]$ and has emerged as one of the most prominent frameworks adopted in the study of motivation and behavioral outcomes. Consistently, research has shown self-efficacy as a key variable within the SCT and is said to be the most powerful factor to consider when predicting behavior [12]. Surprisingly, little research has involved the application of the SCT in its entirety within the PA domain. Of the research that has, only self-efficacy was found to have any predictive value on PA behaviour [14]. Thus, the remainder of this section will discuss the self-efficacy theory and consider its predictive utility and application within the PA domain.

\section{The Self-Efficacy Theory within Physical Activity}

Since its conception over 30 years ago literally hundreds of PA-related research has utilised the construct of self-efficacy as an antecedent, outcome, or process variable when trying to understand the motivational process involved in either sporting or exercise performance [15]. The reason for its popularity is twofold. As discussed, self-efficacy for PA has been shown to predict those individuals who will engage in such endeavours [14] but of greater value is that the construct of self-efficacy comes with specific guidelines for its development. This undoubtedly has appealing qualities for practitioners and researchers which may explain popularity of this construct in understanding PA behavior.

There is a diverse body of literature that has utilised the self-efficacy theory within a variety of health and PA contexts, such as weight loss [16, 17], exercise in older adults [18], and exercise in adolescent girls [14]. Specifically, self-efficacy is thought to influence the goals people set, their ability to persist in the face of obstacles, and their capacity to cope with setbacks and stress and as such, directly influence behavioral engagement. Evidence is now clear in support of this. For instance, Dishman et al. [14] evaluated the effects of a school-based intervention on variables of the SCT, designed to emphasise changes on instruction and the school environment. The authors found that the manipulation of self-efficacy had a direct increase in PA levels among adolescent girls and encouraged the use of selfefficacy as a variable that should be targeted to raise PA levels within this population group. Similarly, self-efficacy for PA has been shown to predict engagement in walking and identify adherers and dropouts in PA interventions $[14,16]$.

While the evidence clearly supports the use of selfefficacy as a powerful predictor of behavior, further research has been suggested, particularly in regards to the measurement of self-efficacy. As proposed within the theory, self-efficacy will vary along the dimensions of magnitude, strength, and generality. Though previous research has measured the strength of self-efficacy [12, 14], few have included measures of magnitude and generality. As such, the predictive utility of self-efficacy may be misinterpreted. For instance, consider the findings of Dishman et al. [14] who noted that increased self-efficacy, albeit small, had a direct effect on increasing PA behavior. The authors included a variety of activities deemed popular with high school girls such as aerobics, weight training, dance, and selfdefence classes but failed to measure the generality of efficacy between each of these situations.

It could be that the level of efficacy for participants was high when performing aerobics but relatively small when performing weight training. Providing an overall measurement of efficacy from the four activities, and not activity-specific measurements, could explain the relatively small increases in self-efficacy noted by the authors. It is clear 
that more work is needed in the assessment of self-efficacy in relation to specific behaviors, particularly if the magnitude of self-efficacy towards a specific activity is to be accurately measured.

In summary, research has found a consistent relationship with efficacy and exercise participation in a variety of contexts. The predictive power and ease of operation has made the self-efficacy theory one of the most consistent predictors of health-related behaviours. What is abundantly clear is that while self-efficacy has been studied extensively, it has usually been incorporated into other behavioral models. Researchers have recognised the predictive utility of selfefficacy and feeling that the predictive power of their own theories may be limited, have incorporated self-efficacy. The TPB is one such exemplar and will now be discussed.

\section{The Theory of Planned Behaviour within Physical Activity}

Identifying the decision making process is at the forefront of research into increasing PA levels. One such model that has been used extensively to understand the influencing factors of adoption, motivation and adherence to PA is The TPB [19]. The development of this theory was built upon previous work, The Theory of Reasoned Action (TRA) [20]. The initial model proposed that performance of volitional behaviors (acting without constraints) such as PA is best predicted from an individual's stated intention to participate in that activity. The authors believed that intention is the most immediate or proximal determinant of behavior influenced by two social cognitive variables, attitude and subjective norm. Despite receiving widespread support in predicating intentions and behaviour across a range of health behaviors including smoking, sexual behavior, and food choice [21], the model was expanded upon through the inclusion of the perceived behavioral control (PBC) construct. Though the theory was initially developed to predict volitional behaviors, the inclusion of PBC is important as it helps identify personal and environmental factors not under complete volitional control. To summarise, propositions of the TPB include the following: (a) individuals will engage in a behavior when they evaluate it positively (attitude), believe that significant others want them to engage in it (subjective norm), and perceive it to be under their control (perceived behavioral control); and (b) strong intention and PBC will increase the likelihood of a behavior.

Evidence to date has shown that both the TRA and the TPB perform well in explaining intentions in a range of populations [22]. Specifically within PA, meta-analytic reviews have been conducted and have consistently found the TPB to be superior in the prediction of behavior over the TRA $[23,24]$. While there is certainly abundant evidence supporting the use of TRA/TPB within the PA domain, it has not been without criticism.

Several conceptual and methodological concerns have emerged regarding the effectiveness of the TRA and TPB for explaining intention and behavior. Firstly, there seems to be discrepancies in the literature regarding measurement of the time interval between intention and behavior. Previous meta-analytic reviews [24] found that the strength of the intention-behavior association did not decline over time which contradicts earlier recommendations that intention should be measured as close in time as possible to the behavior [20]. Recent evidence however suggests that intentionbehavior relationships do weaken over time [25]. In their recent meta-analytic review, Downs and Hausenblas [26] did attempt to address this criticism by reviewing 111 TRA/TPB studies carried out within the exercise or PA domain and specifically examined the predictive utility of the intention-behavior relationship. As the authors predicted, the intention-behavior association was seen to be larger in studies that measured intention and behavior within a 1month period compared to the studies with a time interval greater than 1 month. It seems prudent to suggest that to improve the predictive utility of the intention-behavior association, intention assessment must be measured as close as possible to the commencement of required behaviour.

Secondly, in their meta-analysis, Hagger et al. [23] found that studies with older participants (26 and over) had a stronger intention-behavior association compared to studies with younger participants (25 and under). Without doubt intentions between children and older individuals may differ and this seems to have been overlooked in previous research. When we consider other subject characteristics such as ethnicity and gender it is apparent that these considerations may have been overlooked by previous authors. For those reasons it is crucial that further research determines whether these subject characteristics moderate the effect sizes of the TRA and TPB constructs.

It has been shown that the application of both the TRA/TPB has strong support in the literature and has aided the understanding of the intention behavior relationship. However because of the disparity in the implementation of the theory and the methodological and conceptual critiques proposed, we are left with many unanswered questions. While this theory could be used to guide future interventions, greater care is warranted regarding measurement of its constructs. Nevertheless, it appears that because of the high amount of unexplained variance between intention and behavior associated with the TPB, researchers have tended to rely on other theories when developing PA interventions. As research is ongoing to enhance the predictive validity of the TPB, it is questionable given its ambiguity whether researchers should rely solely upon this theory when constructing future PA interventions.

5.1. Self-Determination Theory. While some individuals participate in regular PA simply for the enjoyment of exercising, others appear to exercise to attain intrinsic or extrinsic rewards such as losing weight, being more attractive, or obtaining recognition from significant others [27]. Previous research has shown that individuals who exercise out of enjoyment rather than being motivated by intrinsic or external rewards are more likely to adhere to a specified exercise programme [28]. Since the goal of health professionals is to promote a continued active lifestyle in individuals 
not currently meeting the current PA recommendations, studying the cognitions that are related to motivation has recently been at the centre of much investigation into understanding how to promote long-term behaviour change. One theory of human motivation that has been applied extensively to the understanding of exercise behaviour is the self-determination theory (SDT) [29].

Although there are many approaches to initiating behaviour change, research has shown that, without successful behavioural intervention, approximately 50\% of individuals who start a PA program will, on average, drop out within the first six months [30]. The SDT, in contrast, focuses on the processes through which a person acquires the motivation for initiating new health-related behaviours and maintaining them over time. The theory assumes that individuals by nature are active, interested, curious, selfmotivated, and eager to succeed. What it also recognises though is that individuals can be alienated or passive and disaffected and accounts for these differences in terms of the types of motivation, which stems from the interaction between individuals inherent active nature and the social environments that either support or thwart that nature [27].

Specifically, the SDT proposes that behavioural regulation towards an activity varies in the extent to which it is autonomous (self-determined), which involves behaving with a full sense of volition and choice, or controlling, which involves behaving with the experience of pressure and demand toward specific outcomes that comes from forces perceived to be external to the self [28]. Furthermore, the theory proposes that individuals have three basic psychological needs, autonomy, competence and relatedness. Autonomy refers to being the perceived origin or source of ones own behaviour, competence, refers to feeling effective in ones ongoing interactions with the social environment and experiencing opportunities of fulfillment, while relatedness refers to feeling connected to others and to have a feeling of belongingness with individuals and the community [27]. The existence of these needs has been proven empirically [31] and is perceived to be essential to all individuals as they can act in an intrinsically motivated fashion towards behaviour because they perceive it as being important in satisfying these psychological needs.

So when these three basic psychological needs are satisfied, an individual's inherent activity will be supported, optimal motivation will be promoted, and positive psychological, developmental, and behavioural outcomes will be produced [27]. Conversely, social environments that thwart satisfaction of these needs yield less optimal forms of motivation and have deleterious effects on a wide variety of well-being outcomes. To conclude, it is evident that the SDT is a dialectic theory which views the environment as nurturing needsatisfaction and motivation.

5.2. Components of the SDT. As an explanation into the motives behind individuals partaking in, and maintaining, an active lifestyle through changes in their behaviour, the SDT has demonstrated exceptional longevity since its initial conception more than three decades ago. Beginning with the basic premise that the most useful theories of motivation would be broad in scope, encompassing a wide range of phenomena, use concepts that have phenomenological or personal meaning for people, be derived using empirical methods and have principles that can be applied across life's domains, [28] the SDT has evolved in the form of mini-theories. So in a sense the SDT is actually a metatheory comprised of subtheories that seek to explain human motivation and behaviour based on individual differences. Rather than being stand-alone theories, the minitheories are readily integrateable with one another in that they all share organismic and dialectical assumptions and all involve the concept of basic psychological needs [27]. Thus, together they constitute the SDT and, when coordinated, cover all types of human behaviour in all domains. We will now briefly discuss the three constituent theories of the SDT.

5.3. Cognitive Evaluation Theory. The cognitive evaluation theory (CET) was the first subtheory to be developed to explain the effects of intrinsic motivation on behaviours and how social contexts affect intrinsic motivation. The theory was formulated to account for reward effects on intrinsic motivation and suggests that autonomy and competence are integral constructs of intrinsic motivation and that contextual events, such as rewards, positive feedback or the imposition of a deadline, are likely to have an affect on an individuals intrinsic motivation towards a behaviour or activity [29].

Research examining the undermining effect and the informational function of the reward in the CET has been extensive [32] although very few have actually been carried out in the domain of exercise and health. A possible explanation for this is that exercise behaviour is unlikely to be viewed as an interesting endeavour and performed solely for extrinsic rewards. This seems plausible as it should be remembered that the CET when formulated was only proposed to apply to behaviours and tasks that are highly interesting rather than to mundane tasks that could be viewed as monotonous and boring and unlikely to be intrinsically motivated [29]. Consequently, the CET cannot explain how uninteresting behaviours can be prompted or more importantly how to promote self-regulation of these behaviours so that individuals will persist over a prolonged period of time. As initial research utilising the CET focused on the intrinsic-extrinsic distinction, it seems that other forms of motivation may be apparent in the control of behaviour in uninteresting behaviours and explains why so few studies have been carried out using the CET in the exercise and health domain.

A further limitation of the CET is that although being identified as an important psychological need, relatedness is not accounted for within the theory [29, 31]. Research has shown that extrinsic rewards often undermine intrinsic motivation, although it is also the case that individuals can feel autonomous while being motivated. In an attempt to explain this and incorporate the basic psychological need of relatedness within the SDT, a second subtheory was proposed. 
5.4. Organismic Integration Theory. The Organismic Integration Theory (OIT) extends the essential distinction between intrinsic and extrinsic motivation in the CET and seeks to explain the motives behind individuals engaging in nonintrinsically motivated behaviours. Based on the basic psychological need of relatedness, it is proposed that individuals will seek satisfactory relationships with others and engage in non-intrinsically motivated behaviours to satisfy relatedness and function effectively in the social world [27]. Accordingly motivation for the behaviour can vary depending on the degree to which the value and regulation of the requested behaviour have been internalized and integrated [27].

Numerous experimental and field studies have examined the correlates and consequences of autonomous and controlled motivation and have consistently demonstrated that autonomous regulation is associated with greater persistence; more positive affect; enhanced performance, especially on heuristic activities; and greater psychological well-being [28]. There have also been studies that have examined the utility of self-determined forms of motivation within an exercise context, demonstrating that autonomous motivation is associated with exercise behavioural engagement and adherence over time [33], exercise intentions [34], and perceived competence [35]. These findings have been supported through a recent meta-analysis of 21 studies which confirmed the simplex-ordered structure of relations among the regulation styles and the effects of these on exercise behaviours and outcomes [36].

5.5. Basic Needs Theory. The final subtheory within the SDT refers to the basic needs theory. This last theory was only recently developed in an attempt by Deci and Ryan, [27] to clarify the somewhat often misunderstood meaning of the basic psychological needs of individuals and their relationship to mental health and well-being. As highlighted previously the three basic needs refer to autonomy, competence, and relatedness. The basic needs theory proposes that when individuals feel as though these three needs are supported, both intrinsic motivation and internalization are supported. Conversely, if the social context inhibits or neglects one of these needs then intrinsic motivation and internalization will be reduced. So, within an exercise-related context, if individuals feel as if a particular behaviour has the potential to fulfil valued goals then participation in that behaviour will increase. With such endeavours the authors propose that the behaviours will become internalised, finally becoming integrated into an individuals set of behaviours that will satisfy the three basic psychological needs.

Understanding the conditions that foster, rather than undermine, these psychological needs has enormous potential for the development of social environments that will promote self-determined motivation, personal development, and well-being. Although it is well documented that the satisfaction of the needs for autonomy, competence, and relatedness will predict health and well-being [27, 28], surprisingly little research has been carried out on the measurement of perceived competence, autonomy, and relatedness in exercise contexts [37]. Given that optimising participant motivation is a central issue in PA interventions and that the satisfaction of the three basic needs is seen as fundamental to individuals approaching behaviours in an intrinsically motivated fashion, it is perplexing to consider why this component within the SDT has been poorly examined in a PA behavioural context.

5.6. Evaluation and Critique of the Self-Determination Theory within Physical Activity. Until recently the vast body of research that has adopted tenants of the SDT within an exercise domain has not been without its limitations. Much of the research that has been carried out in the exercise domain has been methodologically flawed. In their recent meta-analysis Chatzisarantis et al. [36] identified 21 published articles that adopted the Perceived Locus Of Control (PLOC) in the exercise domain and found that, on the whole, findings supported the existence of a selfdetermination continuum. Nevertheless, the authors did question this assumption due to the small number of studies within the meta-analysis and the distinct lack of consistency in the methodological approach used within the studies. Much of the studies seemed to adopt correlational designs rather than experimental designs. Given that researchers can only infer causality when adopting correlational designs it is reasonable to agree with the tentative findings of Chatzisarantis et al. [36] specifically with regards to the process of internalization.

While experimental methods and intervention studies have been extensively adopted by researchers in the sporting domain, until recently very little research had adopted these approaches within an exercise behavioural context. Edmunds et al. [38] though have shown that interventions designed to change motivational regulations in individuals can produce increases in exercise behaviour. Specifically, the researchers examined whether an exercise instructor's teaching style could be manipulated so that it is was perceived by individuals as providing more autonomy support, structure, and interpersonal involvement. In addition the authors also examined the impact of the delivery of the exercise class on the basic psychological needs, autonomous motivation, and behavioural outcomes. Of the two groups that participated in the study, one group acted as a control group whereas in the second group, the treatment group, the instructor focused upon promoting autonomy support by taking the perspective of the exercise class participants into account, acknowledging their feelings and providing them with pertinent information and opportunities for choice.

In comparison to the control group, the individuals within the treatment group reported significantly greater increases in relatedness and competence need satisfaction, positive affect, structure, and interpersonal involvement which corresponded to increased participation and retention rates. The authors concluded that interventions grounded in the SDT could positively influence exercise class participants' behavioural, cognitive, and affective responses to exercise and should be considered when designing future interventions studies. Unfortunately, and as the authors state, this 
study was not without its limitations. Firstly, the investigation only lasted 10 weeks and failed to consider whether this approach would lead to long-term participation. Secondly, a lack of generalizability of the findings is present since only female participants aging between 18 and 53 years were recruited.

Although numerous motivational theories exist, researchers seeking to understand the social conditions that support need satisfaction and subsequent motivation have shown a keen interest in the application of the SDT within health-related exercise contexts. The theoretical framework proposed has been well supported within a variety of contexts, including PA, and is increasingly being used and recommended to guide future intervention and experimental studies. While recent research has supported the application of interventions grounded in SDT [38], it is evident from this discussion that further research is needed within the field of exercise-related behaviour change. The theory has much to offer in terms of predicting behaviour, understanding behavioural mechanisms, and designing appropriate interventions that increase PA participation and adherence. Encouraging individuals to self-regulate and continually form intentions to exercise seem to be the best option to promote adherence to a physically active lifestyle.

\section{The Transtheoretical Model within Physical Activity}

In an attempt to overcome the limitations of social cognitive models, researchers have considered the use of stage-based approaches in behavior change interventions. The most popular stage model applied with a PA context is the TTM [39]. Within the TTM health behavior adoption and maintenance is described as a cyclic process whereby individuals pass through a series of specific stages, each characterised by a particular pattern of psychosocial and behavioral changes. As such, within the TTM, individuals are classified by their readiness to change into one of five stages: precontemplation, contemplation, preparation, action, and maintenance [40]. Though several attempts at change are likely before maintenance is reached, the progression through the process may in fact strengthen behavior change as individuals learn from past regressions [41].

Designing interventions that meet the requirements of individuals based on their stage of change is considered to not only enhance participation and retentions rates but also reduce the resistance of individuals to initiate difficult behaviors [42]. Consequently, much of the PA research that has utilised the TTM in interventions has been delivered in accordance to an individual's stage of change and have found that individuals progress more towards the action and maintenance stages than control participants [43]. Another valuable feature of the TTM is that not only can it provide a framework to categorise individuals into a particular stage of change, but it also indicates how to encourage individuals to change their behaviour and progress through the different stages. Specifically, Prochaska and Marcus [42] propose that each stage is characterised by particular cognitive and psychodynamic variables, which, if targeted, can move individuals into subsequent stages of behavior. The processes of change included in the TTM represents the behavioral or experiential changes used by an individual to modify their experiences.

The body of evidence regarding the effectiveness of TTM based interventions is mixed. In relation to PA, van Sluijs et al. [44] carried out a systematic review of the literature concerning the effect of stages of change-based interventions and its effects on smoking, PA, and dietary behavior. In relation to PA the authors identified 13 randomised control trials (RCTs) that included stage of change-based and behavioral outcomes and found no evidence of an advantageous effect of stage-based interventions as opposed to alternative approaches. This reflects the findings of previous systematic reviews [45].

Here the authors systematically assessed the effectiveness of PA promotional activities that used a stage-based approach in bringing about changes in health-related behavior. Their inclusion criteria of only using RCTs identified seven trials of activity promotion interventions based on the constructs of the TTM. Four of these seven studies found no significant changes in behavioral outcomes, two studies showed mixed results, while only one study showed significant effects in favour of stage-based interventions. The authors did however raise issue with the methodological quality of the studies used highlighting the lack of consistency between interventions and poor appreciation of participant stage classification. The authors deemed the TTM an unsuitable approach for bringing about positive changes in health behavior though they did suggest the need for further research.

Finally, Adams and White [46] reviewed the effectiveness of 16 TTM informed interventions and found that 73 per cent of short-term ( $<6$ month) studies reported a positive effect of TTM studies over "control conditions." The equivalent long-term (>6 months) proportion was 29 per cent. From these findings it could be suggested that stage-based interventions are no more effective than control conditions in promoting long-term adherence to PA. However, Marshall and Biddle [47] in their meta-analysis included 71 published reports that presented empirical data on at least one core construct of the TTM applied to exercise and PA. From their analysis they were able to support the application of the TTM suggesting that the core constructs of the TTM differ across stages with most in the direction predicted by the theory.

There may be numerous reasons why stage-based interventions may sometimes lack effectiveness though three dominant explanations have been suggested. First, a range of evidence has been used not only to evaluate the model but also to develop arguments in the literature [48]. For instance, there seems to be no evidence-based consensus on which criteria should be used for assessing the methodological quality of studies. Whilst some research has utilized an RCT design, others have no control group with much being crosssectional in nature [49]. Consequently, the items included are, to some extent, arbitrarily chosen. Deciding upon which studies are included and excluded in systematic reviews 
therefore can significantly influence the evidence gathered and thus the consensus given.

Secondly, since there are fundamental differences between some health behaviours and the addictive behaviors upon which the model was originally formulated, a lack of evidence may be due to the fact that some behaviors are simply more suitable to stage-based interventions [48]. Given that PA is a multifaceted complex phenomenon, practitioners who are reliant upon models that focus upon just the individual may be underestimating the true complexity of influences upon the individual. There is a wealth of evidence that has shown that other external and social factors, such as age, gender, and socioeconomic status, influence complex behaviors, which the TTM fails to consider $[6,8,50]$. It seems that the reliance upon the TTM by practitioners helps conceal the multifaceted complexities involved in changing complex behaviors.

\section{Emerging Practice: An Ecological Approach}

Within this paper, only a small selection of behavior theories has been discussed. The inclusion of the models in this paper is based on their popularity and application in intervention studies within the PA domain. While all four models have various contrasting features, they also share some core principles. First, all of the models relate predominantly on changing the behavior of the individual and focus less on the environment. Second, the models exist within a positivist and cognitive-rational paradigm where the main focus is to predict and control. As such, the models consider the determinants of specific behaviors as linear and maybe even more importantly phase staged. Meanwhile, Adams and White [51] state that individualized stage-based interventions are not effective in promoting long-term adherence to PA as they oversimplify the individual's ability to make positive changes to their behavior.

Overall, traditional health behavior models leave a suggestion that interventions, programs, activities, and policies can be fully planned and controlled, with predictable outcomes. This clearly does not reflect the real world of health behavior and PA seen from the developments in obesity and overweight rates throughout the world. What has now become apparent within the last twenty years is that changing health behavior is a complex and multifaceted phenomenon that has multiple levels of influences $[8,52]$. With the reliance upon individual psychosocial models it is now clear that such approaches do little to effect change beyond the individual. Since social ecological models of health behavior [8] focus on individual influences as well as social, policy, and environmental factors that may facilitate or inhibit individual behaviour, researchers have now embraced the use of such frameworks to inform their interventions.

\section{Ecological Models of Physical Activity Promotion}

Ecological models profess that individual, interpersonal, organizational, societal, and community factors should be considered when planning and implementing health promotion interventions [8]. When adopting such an approach it is acknowledged that behavior is influenced by multiple levels and, in order to ensure lasting change, appreciation of this is necessary. The use of multilevel ecological approaches is widely accepted to guide public health policy in the United States (US) [53]. Though the use of ecological approaches within health promotion is relatively recent, its application for understanding behavior is not new. The role of the primary contributors to ecological perspectives and its subsequent utility within health research has been identified and discussed previously $[8,54,55]$ and will not be revisited here. Rather, we will identify the challenges of researchers embracing an ecological perspective to inform interventions within the domain of PA behavior.

Though ecological-based multilevel interventions hold great potential for influencing PA, certain issues associated with such an approach need to be acknowledged. For instance, guiding frameworks such as the Social Ecological Theory [8] and the Ecological Model of Health Behavior [50] are useful for considering the behavioral determinants of health broadly. However, these models were devised to provide an overarching framework to guide interventions and their role in advancing the application of ecological perspectives within health behavior should be commended. Nevertheless, these frameworks fail to provide specific mechanisms through which particular influences may interact and influence specific behaviors. Indeed, the lack of specificity and instruction given presents methodological and conceptual challenges that are not apparent when utilising cognitive based models.

Understandably, it could be for this very reason that researchers within the PA domain are still heavily reliant upon cognitive-based theories which provide applicable measures that can be implemented across numerous domains and setting, particularly when one considers the positivist reductionist approach which seems to govern funding bodies and public health policy which advocates the need for identifying causal pathways in health research. When one considers further the traditional desire of inferring causality and solving problems through rational deduction it is unsurprising that there is still reliance from researchers upon traditional linear cognitive paradigms to inform interventions.

With such an approach there is a belief that behavior can be explained as a linear process whereby decisions are planned and actions instinctively ensue. It is apparent that complex behaviors such as PA do not occur in such a way. Instead, behavior is influenced by multiple levels of factors that interact with one another influencing individuals and subsequent behavior. As such it is now becoming accepted that behavior cannot be understood by measuring individual factors alone but rather behavior emerges due to the complex interactions between multiple levels of influences [55-57].

Increasing acceptance of the complexities involved in human behavior renders the current linear phase stage approach to understanding PA behaviors incongruous. From a PA perspective, the complexities involved in understanding behavior can be viewed as a collection of numerous 
determinants whose actions, though unpredictable, impact upon other determinants [6]. Since these determinants of behavior are often nested within numerous levels of influences as outlined by the ecological framework [8], to fully comprehend the impact of individual determinants upon behavior there must be an acceptance of the mediating role of all determinants. Another important premise to acknowledge is that influence of determinants may change over time with a direct influence upon behavior [58]. This is an important constituent to the study of human behavior which is not embraced within the framework of the four main theoretical models most widely utilized within the PA domain.

Rather, the main determinants are believed to influence individuals whereby behavior change occurs in a linear manner [59]. For instance, traditional research paradigms propose that the measurement, and increases, of cognitions such as attitudes, efficacy, beliefs and intentions over time will determine how effective an intervention is for enhancing behavior. This approach however seems flawed as it fails to appreciate the complexities involved in human behavior whereby change often occurs in a nonlinear manner $[59,60]$.

\section{Chaos and Complexity}

Nonlinear dynamical approaches, such as complexity theory and chaos theory, to the study of health behaviours have recently emerged in the health domain literature $[57,59-$ 61]. As these authors contend, this new approach to understanding behavior has stemmed from the limitations inherent in current approaches that are reliant solely upon traditional cognitive rationale paradigms to explain complex behaviours. With such an approach there is a belief that behaviour can be explained as a linear process whereby decisions are planned and actions instinctively ensue. From the previous discussion however, it is apparent that complex behaviours such as PA do not occur in such a way. Instead, behavior is influenced by multiple levels of factors that interact with one another influencing individuals and subsequent behaviour, hence the term, complex behaviours. As such it is now becoming accepted that behavior cannot be understood by measuring individual factors alone but rather behavior emerges due to the complex interactions between multiple levels of influences which is often spontaneous, uncontrolled, and uncertain [56], hence the term chaotic.

Increasing acceptance of the complexities involved in human behavior, for instance, the initiation and/or maintenance of PA, renders the current linear phase stage approach to understanding PA incongruous. The proposed application of the new science of complex adaptive systems with the PA domain $[59,60]$ may however provide a means to better understand the complexities involved in human behavior. Thus, within a complex adaptive system approach human beings can be viewed as being composed of, and operating within, multiple interacting and self-adjusting systems including individual, interpersonal, organizational, societal, and community systems [8, 50, 57]. From a PA perspective, this complex adaptive system can be viewed as a collection of numerous determinants whose actions, though unpredictable, impact upon other determinants. Since these determinants of behaviour are often nested within numerous levels of influences as outlined by the ecological framework, to fully comprehend the impact of individual determinants upon behavior there must be an acceptance of the mediating role of all determinants [62].

Despite gathering consensus that multilevel approaches are required to ensure long-lasting change in behaviour, the field is limited at present due to the dependence upon crosssectional evidence [63]. Of course this new approach to the study of health behavior is in its infancy and as one would expect there is still a great deal to understand regarding the key determinants of behavior worthy of intervening upon. Nevertheless, the PA domain has begun to embrace the need for ecologically informed PA interventions [64-66]. It could be that we are at the embryonic stage of another paradigm shift with the acceptance that behavior change does not occur in a deterministic and linear fashion, but more so through complex interactions between numerous determinants interacting in a nonlinear manner $[59,61]$.

This principle effectively precludes the inference of causality derived from cross sectional analysis. This premise though is not new given that Bronfenbrenner [67] identified these issues more than 60 years ago stating "piecemeal analysis, fixed in time and space, of isolated aspects and attributes is insufficient and even misleading" [67]. Nonlinear dynamical approaches, such as complexity and chaos theory, to the study of health behaviors have recently emerged in the health domain literature [57, 59-61]. The proposed application of the new science of complex adaptive systems within the PA domain may however provide a means to better understand the complexities involved in human behavior. Within a complex adaptive system approach human beings can be viewed as being composed of, and operating within, multiple interacting and self adjusting systems including individual, interpersonal, organizational, societal, and community systems [60]. From a PA perspective, this complex adaptive system can be viewed as a collection of numerous determinants whose actions, though unpredictable, impact upon other determinants. Since these determinants of behaviour are often nested within numerous levels of influences as outlined by the ecological framework, to fully comprehend the impact of individual determinants upon behavior there must be an acceptance of the mediating role of all determinants [62].

Another important premise of complex adaptive systems is that determinants can change which can affect the behavior of individuals over time [57]. This is an important constituent to the study of human behavior and highlights the limitations of current research practices within the PA domain. For instance, despite gathering consensus that multilevel approaches to health behavior consistent with social ecological frameworks are required to ensure substantial changes in health behaviors, the field is limited at present due to the dependence upon cross sectional evidence [63]. In complex systems, behavior is said to emerge from the interaction over time of numerous determinants influencing said behavior in a non linear manner [61]. Traditional 
research paradigms propose that the measurement, and increases, of cognitions such as attitudes, efficacy, beliefs, and intentions over time will determine how effective an intervention is for enhancing health behaviors. This approach however seems flawed as it fails to appreciate the complexities involved in human behavior whereby change often occurs in a nonlinear manner, rather than the linear, deterministic manner proposed by traditional cognitive paradigms [60].

The seminal paper by Resnicow and Vaughan [59] has ignited debate in the literature [68] regarding the usefulness of the prevailing health behavior theories in understanding PA behavior which warrants further comment. Despite disagreements between authors, both contend that there is a need for further research that incorporates nonlinear concepts into future interventions. While this sentiment is shared there is at present a lack of guidance upon how to implement such approaches within a PA context. Nevertheless, previous and future reviews based upon cross sectional research designs can help inform interventions and generate hypotheses and their importance should not be underestimated.

\section{Summary}

Excellent reviews have already taken place which has enhanced our understanding of the key determinants of specific behaviors, within specific contexts $[52,69-72]$. Nevertheless, researchers are faced with the difficult task of developing site- and behavior-specific ecological models. Though daunting the study of human behavior through ecological and complexity theories affords the generation of hypothesis informed through cross sectional evidence. Identifying the key determinants of behavior relative to individual characteristics, contexts, and activities is the first step in devising appropriate interventions. The challenge thereafter is to consider how to monitor the complex interactions that will occur over time within the multiple levels of influence. As such, a unified model of research and practice which integrates both ecological and complexity theories is very much needed. Only then can we begin to understand the role of the multiple influences upon behavior and begin to translate this evidence into future healthenhancing interventions.

\section{Conclusion}

It is now well established that theoretically informed interventions are imperative for successful physical activity promotion. Though only a small selection of behavior theories have been discussed in this paper it is apparent that two major approaches to PA promotion have dominated the literature, one founded on a stage-based model and the other founded on social cognitive principles. While these approaches have greatly enhanced our understanding of the key determinants of PA behavior it is now apparent that behavior is influenced not only through individual level cognitions. It seems that the increasing acceptance of the complexities involved in human behavior renders the current linear phase stage approach to understanding PA behaviours incongruous. What has now become apparent within the last twenty years is that changing health behavior is a complex and multifaceted phenomenon that has multiple levels of influences $[8,52]$. Since ecological approaches towards health behavior focus on individual influences as well as social, policy researchers have now embraced the use of such frameworks to inform their interventions. However, there is a lack of models available that provide specific mechanisms through which particular influences may interact and influence behavior. Researchers are now faced with the daunting task of relying upon detailed cross sectional evidence to develop and test hypothesis in order to enhance further our understanding of the determinants of PA. It seems that the field of PA research is at the embryonic stage of a paradigm shift towards improving our understanding of complex behaviours through the application of complex ecological interventions. Recently the health domain has embraced the use of nonlinear dynamical approaches, such as complexity theory to the study of complex behaviors. In the PA domain, there is no unified model of research and practice which integrates both ecological and complexity theories. Forthcoming PA models should begin to consider the advantages of incorporating the principles of complexity theories into future intervention programmes. This domain of future work promises to be a worthwhile endeavour in tackling current obesity and inactivity levels and should help generate further understanding of the complexities involved in PA behavior.

\section{References}

[1] World Health Organization, "A healthy city is an active city: a physical activity planning guide," 2008.

[2] S. N. Blair and J. N. Morris, "Healthy hearts-and the universal benefits of being physically active: physical activity and health," Annals of Epidemiology, vol. 19, no. 4, pp. 253256, 2009.

[3] J. F. Sallis, R. B. Cervero, W. Ascher, K. A. Henderson, M. K. Kraft, and J. Kerr, "An ecological approach to creating active living communities," Annual Review of Public Health, vol. 27, pp. 297-322, 2006.

[4] S. Sutton, "How does the Health Action Process Approach (HAPA) bridge the intention-behavior gap? An examination of the model's causal structure," Applied Psychology, vol. 57, no. 1, pp. 66-74, 2008.

[5] C. R. Nigg, B. Borrelli, J. Maddock, and R. K. Dishman, "A theory of physical activity maintenance," Applied Psychology, vol. 57, no. 4, pp. 544-560, 2008.

[6] J. F. Sallis, N. Owen, and E. B. Fisher, "Ecological models of health behavior," in Health Behavior and Health Education: Theory, Research and Practice, K. Glanz, B. K. Rimer, and K Viswanath, Eds., pp. 465-485, Jossey-Bass, San Francisco, Calif, USA, 4th edition, 2008.

[7] N. Humpel, N. Owen, and E. Leslie, "Environmental factors associated with adults' participation in physical activity. A review," American Journal of Preventive Medicine, vol. 22, no. 3, pp. 188-199, 2002.

[8] D. Stokols, "Social ecology and behavioral medicine: implications for training, practice, and policy," Behavioral Medicine, vol. 26, no. 3, pp. 129-138, 2000. 
[9] T. K. Scanlan, P. J. Carpenter, G. W. Schmidt, G. W. Schmidt, J. Simons, and B. Keeler, "An introduction to the sport commitment model," Journal of Sport \& Exercise Psychology, vol. 15, no. 1, pp. 1-15, 1993.

[10] C. J. Browning and S. A. Thomas, "Models of behaviour change in health promotion," in An Evidence-Based Handbook for Social and Public Health, C. J. Browning and S. A. Thomas, Eds., pp. 7-15, Elsevier, Scotland, UK, 2005.

[11] A. Bandura, Social Learning Theory, Prentice Hall, Englewood Cliffs, NJ, USA, 1977.

[12] E. McAuley, G. J. Jerome, D. X. Marquez, S. Elavsky, and B. Blissmer, "Exercise self-efficacy in older adults: social, affective, and behavioral influences," Annals of Behavioral Medicine, vol. 25, no. 1, pp. 1-7, 2003.

[13] B. Resnick, M. H. Palmer, L. S. Jenkins, and A. M. Spellbring, "Path analysis of efficacy expectations and exercise behaviour in older adults," Journal of Advanced Nursing, vol. 31, no. 6, pp. 1309-1315, 2000.

[14] R. K. Dishman, R. W. Motl, R. Saunders et al., "Self-efficacy partially mediates the effect of a school-based physical-activity intervention among adolescent girls," Preventive Medicine, vol. 38, no. 5, pp. 628-636, 2004.

[15] M. S. Hagger and N. Chatzisarantis, Social Psychology of Exercise and Sport, Open University Press, Maidenhead, UK, 2005.

[16] E. McAuley, B. Blissmer, J. Katula, and T. E. Duncan, "Exercise environment, self-efficacy, and affective responses to acute exercise in older adults," Psychology and Health, vol. 15, no. 3, pp. 341-355, 2000.

[17] E. McAuley, B. Blissmer, J. Katula, T. E. Duncan, and S. L. Mihalko, "Physical activity, self-esteem, and self-efficacy relationships in older adults: a randomized controlled trial," Annals of Behavioral Medicine, vol. 22, no. 2, pp. 131-139, 2000.

[18] C. B. Dallow and J. Anderson, "Using self-efficacy and a transtheoretical model to develop a physical activity intervention for obese women," American Journal of Health Promotion, vol. 17, no. 6, pp. 373-381, 2003.

[19] I. Ajzen, "The theory of planned behavior," Organizational Behavior and Human Decision Processes, vol. 50, no. 2, pp. 179$211,1991$.

[20] I. Ajzen and M. Fishbein, Understanding Attitudes and Predicting Social Behavior, Prentice Hall, Englewood Cliffs, NJ, USA, 1980.

[21] C. J. Armitage and M. Conner, "Efficacy of the theory of planned behaviour: a meta-analytic review," British Journal of Social Psychology, vol. 40, part 4, pp. 471-499, 2001.

[22] N. L. D. Chatzisarantis, C. Frederick, S. J. H. Biddle, M. S. Hagger, and B. Smith, "Influences of volitional and forced intentions on physical activity and effort within the theory of planned behaviour," Journal of Sports Sciences, vol. 25, no. 6, pp. 699-709, 2007.

[23] M. S. Hagger, N. L. Chatzisarantis, and S. J. Biddle, "A meta-analytic review of the theories of reasoned action and planned behavior in physical activity: predictive validity and the contribution of additional variables," Journal of Sport and Exercise Psychology, vol. 24, pp. 3-32, 2002.

[24] H. A. Hausenblas, A. V. Carron, and D. E. Mack, "Application of the theories of reasoned action and planned behavior to exercise behavior: a meta-analysis," Journal of Sport and Exercise Psychology, vol. 19, no. 1, pp. 36-51, 1997.

[25] N. L. D. Chatzisarantis, M. S. Hagger, S. J. H. Biddle, and B. Smith, "The stability of the attitude-intention relationship in the context of physical activity," Journal of Sports Sciences, vol. 23, no. 1, pp. 49-61, 2005.

[26] D. S. Downs and H. A. Hausenblas, "The theories of reasoned action and planned behavior applied to exercise: a metaanalytic update," Journal of Physical Activity and Health, vol. 2, no. 1, p. 76, 2005.

[27] E. L. Deci and R. M. Ryan, "The "what" and "why" of goal pursuits: human needs and the self-determination of behavior," Psychological Inquiry, vol. 11, no. 4, pp. 227-268, 2000.

[28] E. L. Deci and R. M. Ryan, "Facilitating optimal motivation and psychological well-being across life's domains," Canadian Psychology, vol. 49, no. 1, pp. 14-23, 2008.

[29] E. L. Deci and R. M. Ryan, Intrinsic Motivation and SelfDetermination in Human Behavior, Plenum Press, New York, NY, USA, 1985.

[30] R. K. Dishman and J. Buckworth, "Increasing physical activity: a quantitative synthesis," Medicine and Science in Sports and Exercise, vol. 28, no. 6, pp. 706-719, 1996.

[31] K. M. Sheldon, A. J. Elliot, Y. Kim, and T. Kasser, "What is satisfying about satisfying events? Testing 10 candidate psychological needs," Journal of Personality and Social Psychology, vol. 80, no. 2, pp. 325-339, 2001.

[32] E. L. Deci, R. Koestner, and R. M. Ryan, "A meta-analytic review of experiments examining the effects of extrinsic rewards on intrinsic motivation," Psychological Bulletin, vol. 125, no. 6, pp. 627-668, 1999.

[33] N. L. D. Chatzisarantis, M. S. Hagger, S. J. H. Biddle, and C. Karageorghis, "The cognitive processes by which perceived locus of causality predicts participation in physical activity," Journal of Health Psychology, vol. 7, no. 6, pp. 685-699, 2002.

[34] M. S. Hagger, N. L. D. Chatzisarantis, V. Barkoukis et al., "Cross-cultural generalizability of the theory of planned behavior among young people in a physical activity context," Journal of Sport and Exercise Psychology, vol. 29, no. 1, pp. 220, 2007.

[35] M. Goudas, S. J. Biddle, and K. Fox, "Perceived locus of causality, goal orientations, and perceived competence in school physical education classes," The British Journal of Educational Psychology, vol. 64, pp. 453-463, 1994.

[36] N. L. D. Chatzisarantis, M. S. Hagger, S. J. H. Biddle, B. Smith, and J. C. K. Wang, "A meta-analysis of perceived locus of causality in exercise, sport, and physical education contexts," Journal of Sport and Exercise Psychology, vol. 25, no. 3, pp. 284306, 2003.

[37] R. J. Vallerand and G. F. Losier, "An integrative analysis of intrinsic and extrinsic motivation in sport," Journal of Applied Sport Psychology, vol. 11, no. 1, pp. 142-169, 1999.

[38] J. Edmunds, N. Ntoumanis, and J. L. Duda, “Testing a selfdetermination theory-based teaching style intervention in the exercise domain," European Journal of Social Psychology, vol. 38, no. 2, pp. 375-388, 2008.

[39] J. O. Prochaska and C. C. DiClemente, The Transtheoretical Approach: Crossing Traditional Boundaries of Therapy, Dow Jones-Irwin, Homewood, Ill, USA, 1984.

[40] B. H. Marcus and L. Forsyth, Motivating People to be Physically Active, Human Kinetics Books, Champaign, Ill, USA, 2003.

[41] B. H. Marcus and L. R. Simkin, "The stages of exercise behavior," Journal of Sports Medicine and Physical Fitness, vol. 33, no. 1, pp. 83-88, 1993.

[42] J. O. Prochaska and B. Marcus, "The transtheoretical model: application to exercise," in Advances in Exercise Adherence, R. K. Dishman, Ed., pp. 161-180, Human Kinetics Books, Champaign, Ill, USA, 1994. 
[43] A. F. Kirk, N. Mutrie, P. D. MacIntyre, and M. B. Fisher, "Promoting and maintaining physical activity in people with type 2 diabetes," American Journal of Preventive Medicine, vol. 27, no. 4, pp. 289-296, 2004.

[44] E. M. F. van Sluijs, M. N. M. van Poppel, J. W. R. Twisk, and W. van Mechelen, "Physical activity measurements affected participants' behavior in a randomized controlled trial," Journal of Clinical Epidemiology, vol. 59, no. 4, pp. 404-411, 2006.

[45] R. P. Riemsma, J. Pattenden, C. Bridle et al., "A systematic review of the effectiveness of interventions based on a stagesof-change approach to promote individual behaviour change," Health Technology Assessment, vol. 6, no. 24, pp. 1-231, 2002.

[46] J. Adams and M. White, "Are activity promotion interventions based on the transtheoretical model effective? A critical review," British Journal of Sports Medicine, vol. 37, no. 2, pp. 106-114, 2003.

[47] S. J. Marshall and S. J. H. Biddle, "The transtheoretical model of behavior change: a meta-analysis of applications to physical activity and exercise," Annals of Behavioral Medicine, vol. 23, no. 4, pp. 229-246, 2001.

[48] C. Bridle, R. P. Riemsma, J. Pattenden et al., "Systematic review of the effectiveness of health behavior interventions based on the transtheoretical model," Psychology and Health, vol. 20, no. 3, pp. 283-301, 2005.

[49] R. West, "Time for a change: putting the transtheoretical (Stages of Change) model to rest," Addiction, vol. 100, no. 8, pp. 1036-1039, 2005.

[50] K. R. McLeroy, D. Bibeau, A. Steckler, and K. Glanz, "An ecological perspective on health promotion programs," Health Education Quarterly, vol. 15, no. 4, pp. 351-377, 1988.

[51] J. Adams and M. White, "Why don't stage-based activity promotion interventions work?" Health Education Research, vol. 20, no. 2, pp. 237-243, 2005.

[52] J. F. Sallis and K. Glanz, "The role of built environments in physical activity, eating, and obesity in childhood," The Future of Children, vol. 16, no. 1, pp. 89-108, 2006.

[53] US Department of Health and Human Services, Healthy People 2010, US Department of Health and Human Services, Wsahington, DC, USA, 2000.

[54] J. F. Sallis and N. Owen, "Ecological models," in Health Behavior and Health Education: Theory, Research and Practice, K. Glanz, F. M. Lewis, and B. K. Rimer, Eds., pp. 304-424, Jossey-Bass, San Francisco, Calif, USA, 1997.

[55] L. McLaren and P. Hawe, "Ecological perspectives in health research," Journal of Epidemiology and Community Health, vol. 59, no. 1, pp. 6-14, 2005.

[56] U. Bronfenbrenner, Making Human Beings Human : Bioecological Perspectives on Human Development, Sage, Thousand Oaks, Calif, USA, 2005.

[57] T. Wilson, T. Holt, and T. Greenhalgh, "Complexity science: complexity and clinical care," British Medical Journal, vol. 323, no. 7314, pp. 685-688, 2001.

[58] P. E. Plsek and T. Wilson, "Complexity science: complexity, leadership, and management in healthcare organisations," British Medical Journal, vol. 323, no. 7315, pp. 746-749, 2001.

[59] K. Resnicow and R. Vaughan, "A chaotic view of behavior change: a quantum leap for health promotion," International Journal of Behavioral Nutrition and Physical Activity, vol. 3, article 25, 2006.

[60] K. Resnicow and S. E. Page, "Embracing chaos and complexity: a quantum change for public health," American Journal of Public Health, vol. 98, no. 8, pp. 1382-1389, 2008.
[61] P. E. Plsek and T. Greenhalgh, "Complexity science: the challenge of complexity in health care," British Medical Journal, vol. 323, no. 7313, pp. 625-628, 2001.

[62] A. E. Bauman, "The physical environment and physical activity: moving from ecological associations to intervention evidence," Journal of Epidemiology and Community Health, vol. 59, no. 7, pp. 535-536, 2005.

[63] J. P. Elder, L. Lytle, J. F. Sallis et al., "A description of the socialecological framework used in the trial of activity for adolescent girls (TAAG)," Health Education Research, vol. 22, no. 2, pp. 155-165, 2007.

[64] P. J. Naylor, H. M. Macdonald, J. A. Zebedee, K. E. Reed, and H. A. McKay, "Lessons learned from Action Schools! BC_-an "active school" model to promote physical activity in elementary schools," Journal of Science and Medicine in Sport, vol. 9, no. 5, pp. 413-423, 2006.

[65] D. S. Buchan, S. Ollis, N. E. Thomas, and J. S. Baker, "The influence of a high intensity physical activity intervention on a selection of health related outcomes: an ecological approach," BMC Public Health, vol. 10, no. 1, article 8, 2010.

[66] P. J. Naylor and H. A. McKay, "Prevention in the first place: schools a setting for action on physical inactivity," British Journal of Sports Medicine, vol. 43, no. 1, pp. 10-13, 2009.

[67] U. Bronfenbrenner, "A constant frame of reference for sociometric research: part II. Experiment and inference," Sociometry, vol. 7, pp. 40-75, 1944.

[68] J. Brug, "Order is needed to promote linear or quantum changes in nutrition and physical activity behaviors: a reaction to "a chaotic view of behavior change" by Resnicow and Vaughan," International Journal of Behavioral Nutrition and Physical Activity, vol. 3, article 29, 2006.

[69] J. F. Sallis, J. J. Prochaska, and W. C. Taylor, "A review of correlates of physical activity of children and adolescents," Medicine and Science in Sports and Exercise, vol. 32, no. 5, pp. 963-975, 2000.

[70] S. G. Trost, N. Owen, A. E. Bauman, J. F. Sallis, and W. Brown, "Correlates of adults' participation in physical activity: review and update," Medicine and Science in Sports and Exercise, vol. 34, no. 12, pp. 1996-2001, 2002.

[71] B. E. Saelens, J. F. Sallis, and L. D. Frank, "Environmental correlates of walking and cycling: findings from the transportation, urban design, and planning literatures," Annals of Behavioral Medicine, vol. 25, no. 2, pp. 80-91, 2003.

[72] N. W. Burton, G. Turrell, B. Oldenburg, and J. F. Sallis, "The relative contributions of psychological, social, and environmental variables to explain participation in walking, moderate-, and vigorous-intensity leisure-time physical activity," Journal of Physical Activity and Health, vol. 2, no. 2, pp. 181-196, 2005. 


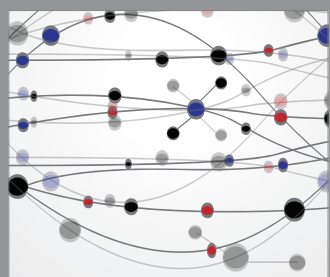

The Scientific World Journal
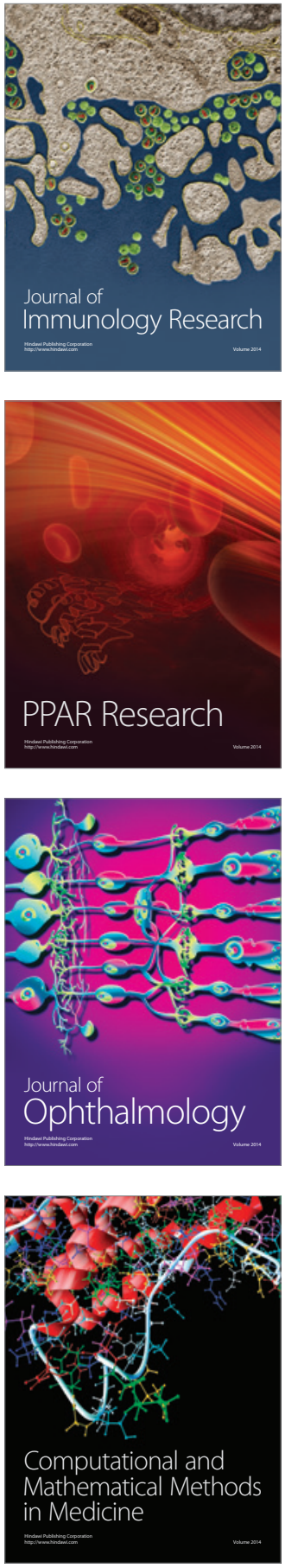

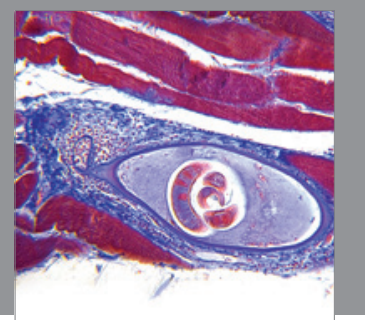

Gastroenterology

Research and Practice
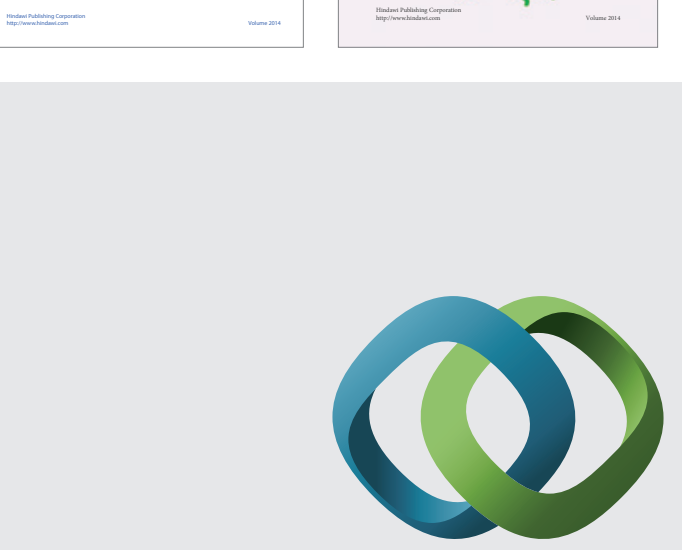

\section{Hindawi}

Submit your manuscripts at

http://www.hindawi.com
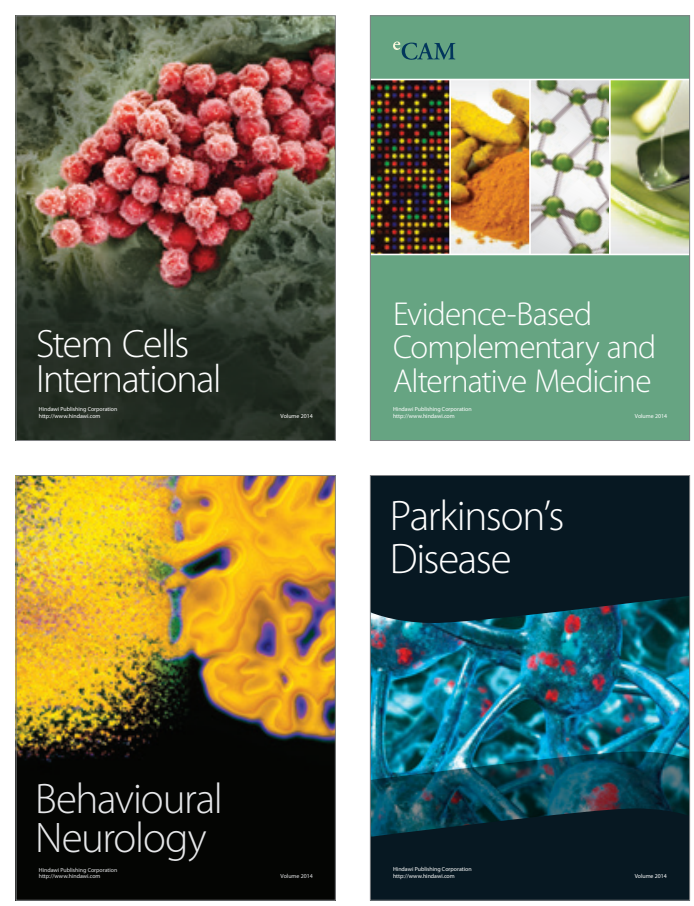

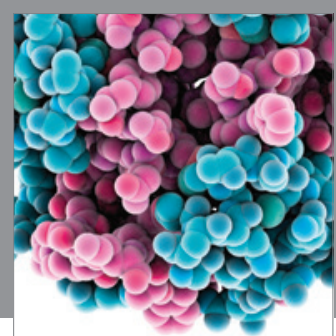

Journal of
Diabetes Research

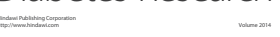

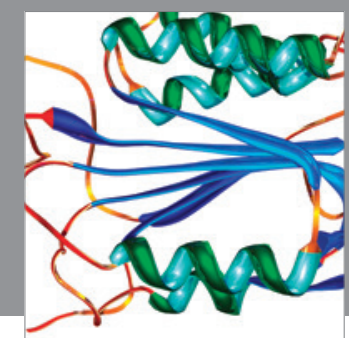

Disease Markers
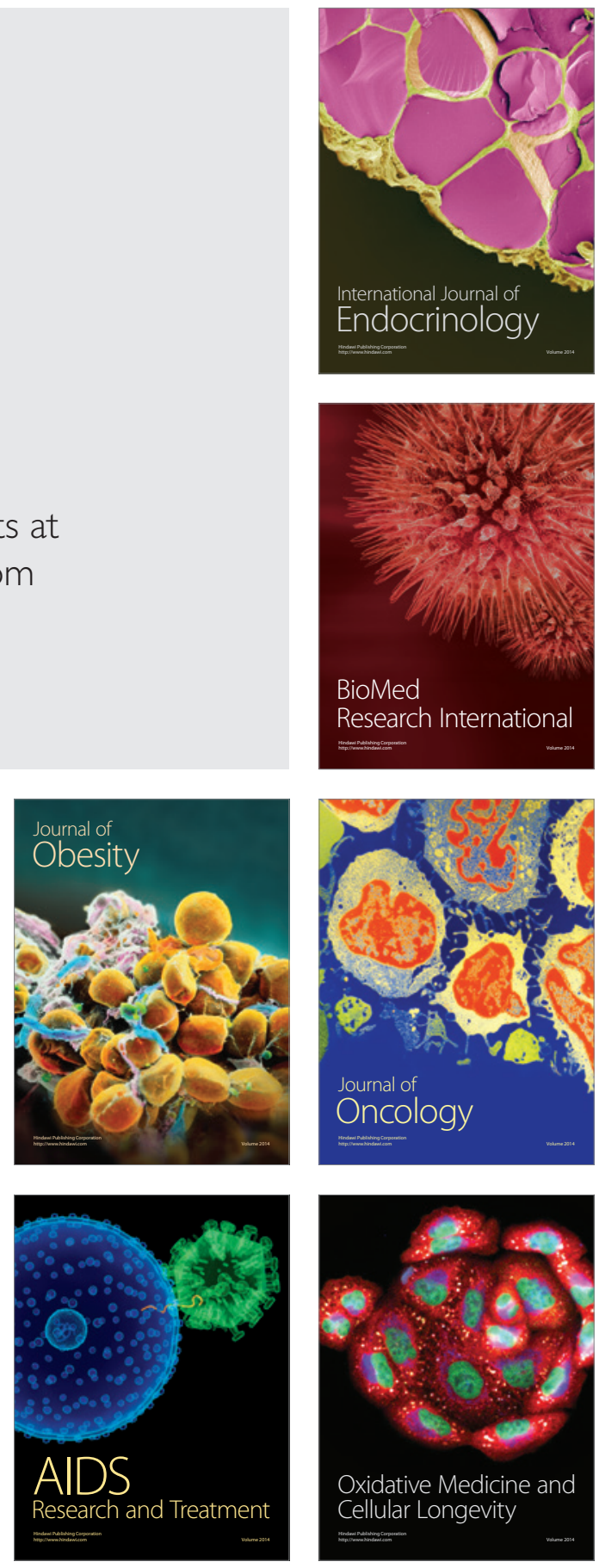